\title{
Identification of PICK1 PDZ-Domain antagonists: Pharmacophore- based virtual screening, molecular docking, and molecular dynamic simulation analyses
}

\author{
Shravan B. Rathod \\ PICK1 (protein interacting with C kinase-1) plays a key role in the regulation of intracellular trafficking of AMPA GluA2 subunit \\ that is linked with synaptic plasticity. PICK1 is a scaffolding protein and binds numerous proteins through its PDZ domain. \\ Research showed that synaptic plasticity is altered upon disrupting the GluA2-PDZ interactions. Inhibiting PDZ and GluA2 \\ binding lead to beneficial effects in the cure of neurological diseases thus, preventing PDZ-GluA2 binding is thought to novel \\ therapeutic target in such diseases. To target this, generally, peptides were synthesized and tested. Though small organic \\ molecules have been targeted to prevent these interactions, the number of such molecules is inadequate. Thus, in this study, \\ ten molecular libraries containing large numbers of molecules were screened against the PDZ domain using pharmacophore- \\ based virtual screening to find the best hits for the PDZ domain. Molecular docking and molecular dynamic simulation studies \\ revealed that two hits (Hit_I and Hit_III) show efficient binding to the PDZ domain. This study suggests that tested hits may \\ have potency against the PDZ domain and can be considered effective to treat neurological disorders.
}

\section{Introduction}

Protein interacting with $C$ kinase-1 (PICK1) is a membrane protein and present in a wide array of species from $C$. elegans to humans. It is abundantly expressed in brain and testis tissues in humans. In the cellular region, it is found at the perinuclear site and the neural presynaptic and postsynaptic sections of the central nervous system (CNS). ${ }^{1}$ PICK1 contains two vital domains, membrane binding N-BAR (bin/amphiphysin/rvs) and PDZ (PSD-95/Dlg/ZO1) which binds to the PDZ motifs of other proteins. $^{2-3}$ The PDZ domain of PICK1 interacts with various transport proteins, neurotransmitter receptors, and other enzymes. ${ }^{1,4}$ Since PICK1 is involved in the regulation of proteins that are linked with neuropsychiatric and neurological conditions, it can be considered a potential target for novel therapeutics. $^{5}$

The disruption of protein-protein interactions implicated in cell-cell adhesion, cell death, signal transduction, and other biomolecular processes by small organic molecules can solve many biological challenges for numerous diseases. ${ }^{6-8}$ PDZ domain is involved in therapeutically targeted protein-protein interactions ${ }^{9-11}$ and it helps trafficking and large assembly forming proteins through providing scaffolding sites inside the cell. This domain identifies the $\mathrm{C}$-terminal region of interacting proteins to facilitate further functions and it is highly expressed in eukaryotes. ${ }^{12-14}$ Research shows that PICK1 plays a vital role in different forms of synaptic plasticity ${ }^{15-18}$ involving LTD (longterm depression) and LTP (long-term potentiation) through its binding with $\alpha$-amino-3-hydroxy-5-methyl-isoxazole-4propionic acid (AMPA) receptors and, it has been also reported therapeutic target for curing pain, ${ }^{19}$ brain ischemia, ${ }^{20-21}$ and cocaine addiction. ${ }^{22}$ glutamate receptor (GluA2) subunit of AMPA interacts with the PDZ domain of PICK1 through its Cterminal which is necessary for the AMPA internalization in the cell. ${ }^{23}$ This enhanced internalization of AMPA through GluA2 mediated mechanism leads to further synaptic depression by amyloid-beta $(A \beta)$ which results in a reduction of dendritic spine density. ${ }^{24}$ Study showed that small molecule inhibitors mitigate the effects of $A \beta$ on synapse through disrupting PDZ-GluA2 interactions thus, it indicates the role of these interactions is vital to $A \beta$ effects on synaptic functions. ${ }^{25}$ Hence, preventing the interactions between AMPA and PICK1 by small molecules can be considered a significant approach to treat diseases and disorders.

To target the PDZ domain, researchers have designed and investigated short peptides, modified peptides, cyclic peptides, and peptidomimetics but due to their less cell permeability, results were not quite promising. ${ }^{26}$ Moreover, Bach and his team synthesized dimeric peptide that showed high binding affinity $(K i=4.6 \mathrm{nM})$ with the PDZ domain of PICK1. But, these inhibitors have poor potency, less selectivity, and distribution problems. ${ }^{27}$ To overcome these challenges, researchers have started to synthesize non-peptide small organic molecules against the PDZ domains.5,28-30 Thorsen et al. also used fluorescence polarization (FP) based screening of 43,380 small organic molecules and got promising results targeting PDZ domain. ${ }^{31}$

We still have a less number of PDZ domain inhibiting candidates compared to the availability of small molecule

Department of Chemistry, Smt. S. M. Panchal Science College, Talod, Gujarat, India

Email: shravanathorizon93@gmail.com

ORCID iD: http://orcid.org/0000-0002-1870-2508 


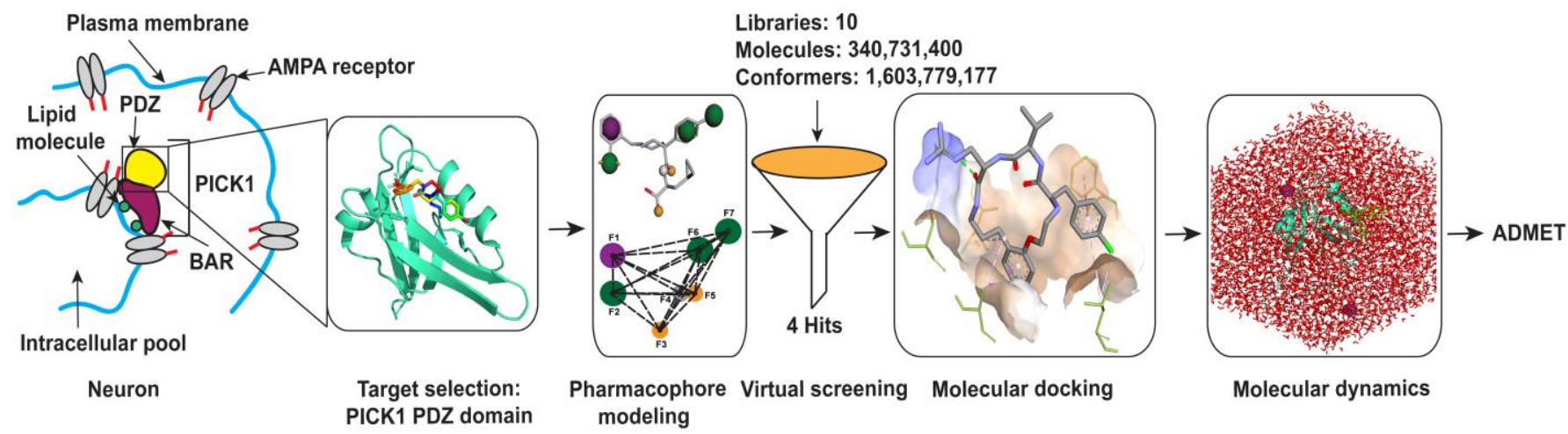

Fig. 1 Methodology schema used in this study to identify the best hits against the PICK1 PDZ domain. PICK1 PDZ domain with its inhibitor BQA was retrieved from the PDB database. Then, PDZ domain and BQA were uploaded to the Pharmit, a pharmacophore-based virtual screening web server. Pharmacophore was constructed using seven features of the BQA inhibitor. Then, ten molecular libraries were screened against the PDZ domain. Obtained four best hits were further analyzed for the binding affinity towards the PDZ domain using DockThor molecular docking web server. Finally, all-atom $50 \mathrm{~ns}$ molecular dynamic simulation was performed using the GROMACS 2020.1 tool to investigate the dynamic behavior of protein-ligand complexes along with control (BQA) complex.

databases. Thus, in this present study, I utilized computer-aided drug design (CADD) tools to screen a vast number of small organic molecules and their conformers to identify potential hits for the PDZ domain. Computational analysis spotted four hits that showed efficient binding towards PDZ and, they significantly decrease the interactions between GluA2 and PDZ domain. Fig. 1 represents the workflow of present study.

\section{Materials and methods}

\section{Protein preparation}

The crystal structure of PICK1 PDZ domain (PDB: 6AR4 ${ }^{27}$ ) with its inhibitor (BQA: N-[4-(4-bromophenyl)-1- $\{[2-$ (trifluoromethyl)phenyl]methyl\}piperidine-4-carbonyl]-3cyclopropyl-L-alanine) was retrieved from the protein data bank (PDB) (https://www.rcsb.org/). The crystal structure of the PICK1 PDZ domain is a dimeric form so, one monomer with its ligand and solvent molecules were deleted from the structure during protein preparation using PyMOL v2.4.1 (Schrodinger LLC., 2010). Additionally, the missing residues (a.a.: 1-18 \& 105109) were added using Modeller ${ }^{32}$ and implicit hydrogens were added in PyMOL.

\section{Structure-based pharmacophore modeling and virtual screening}

Pharmacophore-based small molecule virtual screening was carried out using Pharmit web server ${ }^{33}$ available at http://pharmit.csb.pitt.edu/. For the construction of the pharmacophore model, a prepared protein with its BQA ligand was uploaded to the server. Out of nine features, one feature (hydrogen acceptor) at the carboxylic acid group of BQA was excluded, and remaining parameters were kept unchanged for the pharmacophore modeling. This pharmacophore was further utilized for virtual screening of ten chemical libraries with having $340,731,400$ molecules and 1,603,779,177 conformers (Table 1). After the virtual screening, four hits (RMSD $\leq 4.5 \AA$ ) were identified and their structures (SDF format) were saved for further analysis.

\section{Ligand preparation}

Identified four hits in the virtual screening were opened in Avogadro $^{34}$ and, implicit hydrogens were added to the structures. Further, energy minimization (MMFF94s force field $^{35}$ \& steepest descent algorithm ${ }^{36}$ ) was performed using Auto Optimization Tool in Avogadro. Optimized structures were saved as Sybyl Mol2 format for molecular docking and dynamics analysis.

\begin{tabular}{|c|c|c|}
\hline Library & Molecules & Conformers \\
\hline MCULE-ULTIMATE & $126,471,502$ & $378,880,344$ \\
\hline PubChem & $93,067,404$ & $450,708,705$ \\
\hline ChemSpace & $50,181,678$ & $250,205,463$ \\
\hline MCULE & $45,045,153$ & $222,427,706$ \\
\hline ZINC & $13,190,317$ & $123,399,574$ \\
\hline MolPort & $7,719,859$ & $110,832,826$ \\
\hline LabNetwork & $1,794,286$ & $22,051,020$ \\
\hline CHEMBL25 & $1,752,844$ & $23,136,925$ \\
\hline ChemDiv & $1,456,120$ & $21,562,497$ \\
\hline $\mathrm{NCl}$ Open Chemical Repository & 52,237 & 574,117 \\
\hline
\end{tabular}

\section{Molecular docking}

To investigate the binding affinity of previously obtained four hits (Hit_I, Hit_II, Hit_III, and Hit_IV) along with control (BQA), molecular docking of PICK1 PDZ domain with these small organic molecules was performed at a widely used flexible docking web server, DockThor ${ }^{37}$ (https://dockthor.Incc.br/v2/). To run molecular docking, prepared protein (PDB: 6AR4) and hits were uploaded to the server. Then, a predefined binding

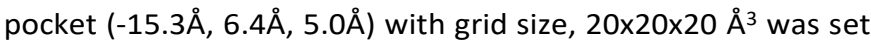
and default options (a. 12 docking runs, b. 500,000 evaluations per docking run, c. population of 750 individuals, and $d$. maximum of 20 cluster leaders on each docking run) were kept unchanged. For each run, DockThor gives the best pose based on the binding affinity and clustering probability of ligand. Results were downloaded for further analysis. 2D and 3D interactions were obtained using Discovery Studio v.20.1 


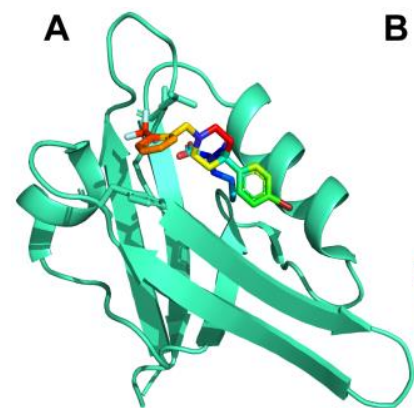

F

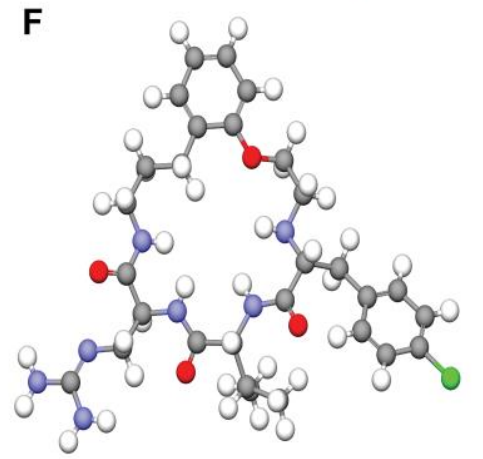

Hit_I

J

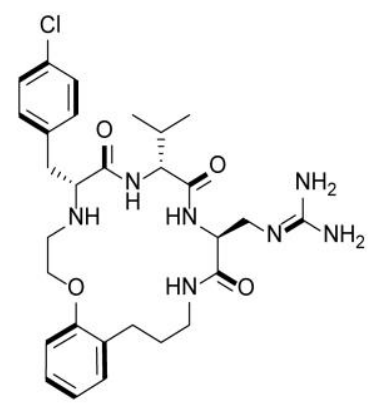

Hit_I

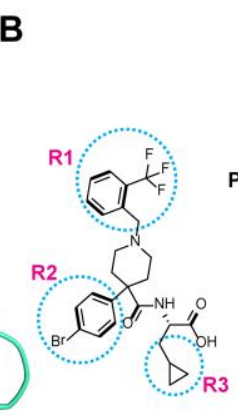

G

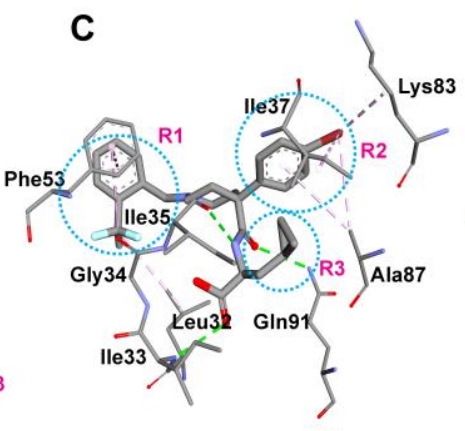

H

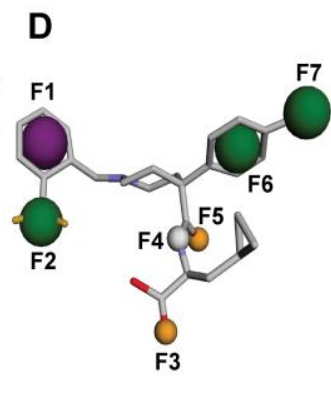

E

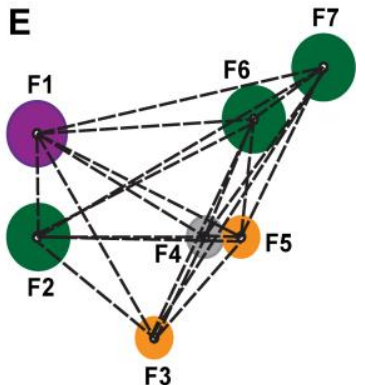

I

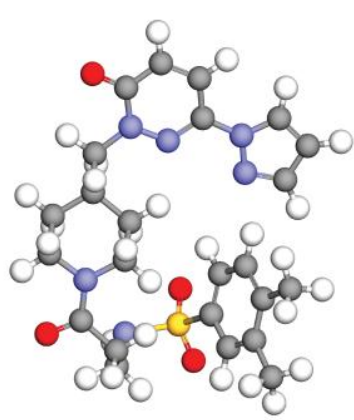

Hit_II

K<smiles>Cc1ccc(S(=O)(=O)NCCC(=O)N2CCC(Cn3nc(-n4cccn4)ccc3=O)CC2)cc1C</smiles>

Hit_II

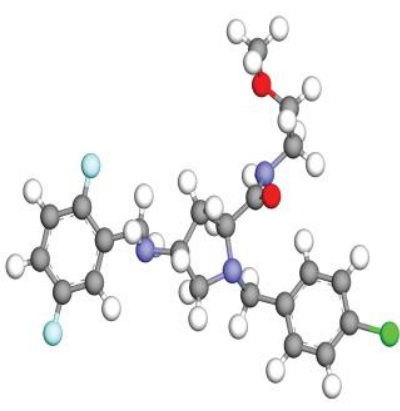

Hit_III

L

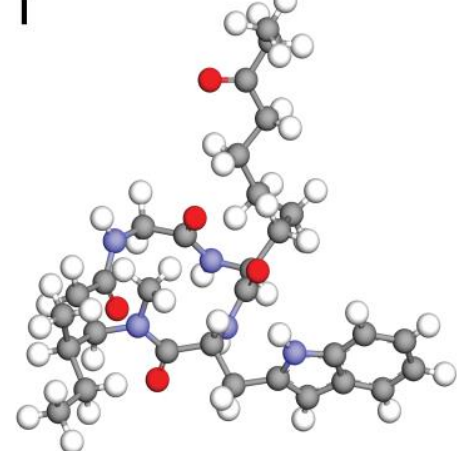

Hit_IV

M<smiles>COCCNC(=O)C1C[C@@H](NCc2cc(F)ccc2F)CN1Cc1ccc(Cl)cc1</smiles>

Hit_III

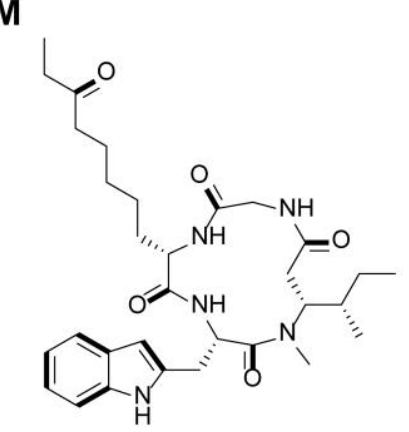

Hit_IV

Fig. 2 Pharmacophore modeling and 2D-3D molecular structures of identified four hits. (A) Crystal structure of PICK1 PDZ domain with BQA inhibitor (PDB: 6AR4). (B) 2D molecular structure of BQA. Ocean blue dashed circles indicate the three hydrophobic pockets, R1, R2, and R3 in BQA. (C) 3D molecular structure of pharmacophore surrounded with the interacting amino acid residues of PDZ domain. (D) Pharmacophore model constructed at Pharmit web server. (E) 3D spatial distribution of the seven pharmacophore features. Distances between features; (1) F1-F2: 2.78 $\AA$ (2) F1-F3:9.03 $\AA$ (3) F1-F4: 7.50 $\AA$ (4) F1-F5: 7.20 $\AA$ (5) F1-F6: 8.78 $\AA$ (6) F1-F7: 11.85

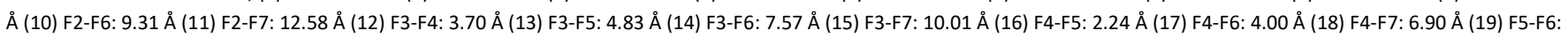
$4.55 \AA ̊$ (20) F5-F7: $7.64 \AA$, and (21) F6-F7: $3.23 \AA \AA$. (F-I) 3D molecular structures of obtained four hits, Hit_I (CHEMBL232154), Hit_II (KWFSWQGHHVLWDM-UHFFFAOYSA-N), Hit_III (MolPort-005-050-255), and Hit_IV (PubChem-46907406) respectively. (J-M) 2D molecular structures of obtained four hits, Hit_I (CHEMBL232154), Hit_II (KWFSWQGHHVLWDMUHFFFAOYSA-N), Hit_III (MolPort-005-050-255), and Hit_IV (PubChem-46907406) respectively.

(BIOVIA, Dassault Systèmes, Discovery Studio Viualizer, 20.1.0.19295, San Diego: Dassault Systèmes, 2020.)

\section{Molecular dynamic (MD) simulation}

To probe the effects of ligand on protein dynamics, molecular dynamic simulation was performed using GROMACS 2020.1 version $^{38}$ on Linux (Ubuntu 2020.1-1) system. MD simulations were performed for apo form (PDZ), control (PDZ-BQA), and four hits (PDZ-Hit_I to Hit_IV). MD simulation was carried out using CHARMM36 force field ${ }^{39-40}$ updated version (charmm36feb2021) and TIP3P water model. ${ }^{41}$ CHARMM general force field (CGenFF) ${ }^{42-43}$ (https://cgenff.umaryland.edu/) was employed to build ligand topologies. Protein and its complexes were placed in the center of the dodecahedron box with a $10 \AA$ from the box edges. Positive $\left(\mathrm{Na}^{+}\right)$and negative $\left(\mathrm{Cl}^{-}\right)$ions were added by substituting solvent molecules to the system wherever they were needed to neutralize the whole system. To reduce steric clashes into the system, energy minimization was performed using the steepest descent algorithm ${ }^{36}$ with Verlet cut-off scheme ${ }^{44}$ and particle mesh ewald (PME) ${ }^{45}$ long-range electrostatic. During energy minimization, a maximum number of steps (nsteps) were 50,000, and system approaching minimum energy was $10 \mathrm{~kJ} / \mathrm{mol}$. Further, NVT and NPT based equilibrations were carried out at $300 \mathrm{~K}$ temperature and for 100 ps (50,000 steps) with a 2 fs time step gap. Finally, MD simulation was performed for 50 ns using leap-frog integrator, ${ }^{46}$ Verlet cut-off scheme, and PME at $300 \mathrm{~K}$ (modified Berendsen thermostat ${ }^{47}$ ) and 1 bar pressure (Parrinello-Rahman 
method ${ }^{48}$ ). All the bonds were constrained with LINCS (linear constraint solver) algorithm. ${ }^{49}$ Required Python script and CHARMM36 force field file were downloaded from the MacKerell lab website (http://mackerell.umaryland.edu/charmm_ff.shtml\#gromacs). MD trajectories were analyzed for various parameters such as root mean square deviation (RMSD), root mean square fluctuation (RMSF), solvent accessible surface area (SASA), radius of gyration $(\mathrm{Rg})$, and intramolecular hydrogen bonds in PDZ and intermolecular hydrogen bonds in the complexes.

\section{Results and discussion}

\section{Catalytic site of PICK1 PDZ domain}

The interactions of the PDZ domain with the C-termini of NMDA (N-methyl-D-aspartate) and with the AMPA GluA2 subunit are thought to play central role in synaptic plasticity. ${ }^{50}$ Lin et al. ${ }^{27}$ have solved the crystal structure of the PICK1 PDZ domain with its inhibitor ( $B Q A)$ and they observed that inhibitor binds to the AMPA GluA2 subunit binding pocket of the PDZ domain. BQA has three main hydrophobic regions (Fig. 1B), which are designated by $R 1, R 2$, and $R 3$. These three sites show dominant interactions with the different amino acid residues of the PDZ domain. Fig. 3 illustrates the $\mathrm{H}$-bond and hydrophobic-

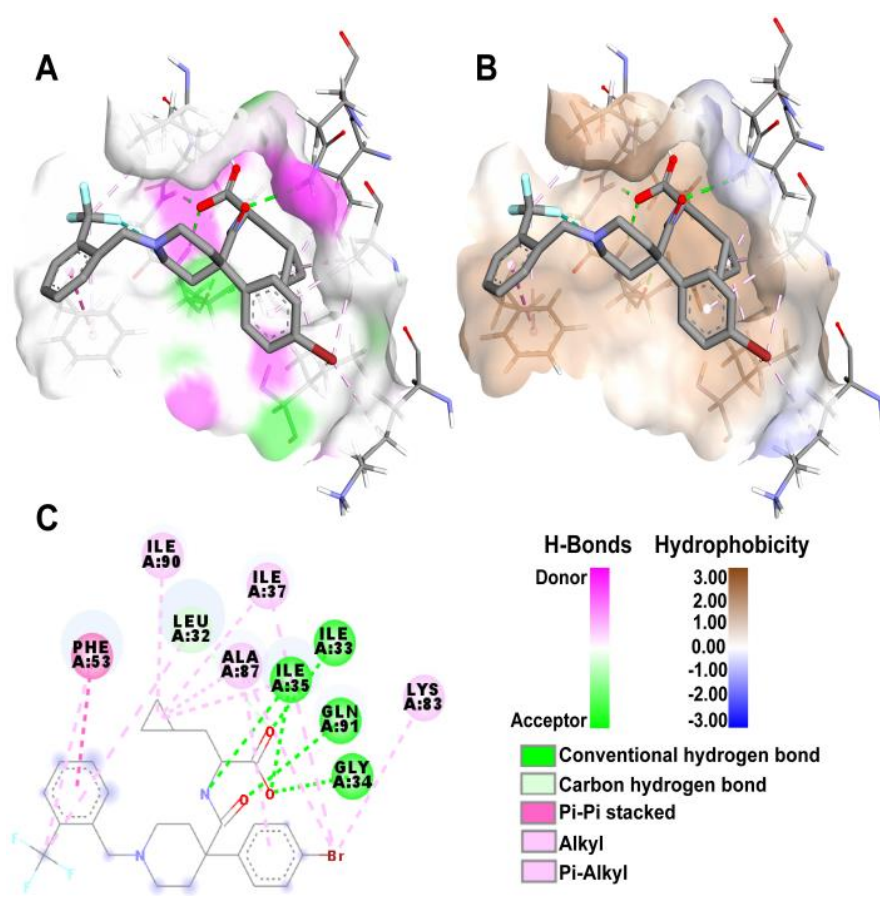

Fig.3 BQA binding pose inside the PDZ domain and 2D interactions. (A) Hydrogen bond surface representation (B) Hydrophobic surface representation, and (C) 2D interactions.

Table 2 Docking results of hits and control (BQA) with the PICK1 PDZ domain calculated at DockThor web server.

\begin{tabular}{|c|c|c|c|c|c|c|c|c|}
\hline Code & ID & $\begin{array}{l}\text { Molecular } \\
\text { Formula }\end{array}$ & $\begin{array}{c}\text { Molecular } \\
\text { Weight } \\
\text { (g/mol) }\end{array}$ & $\begin{array}{l}\text { Binding } \\
\text { affinity } \\
\text { (kcal/mol) }\end{array}$ & $\begin{array}{c}\text { Total } \\
\text { energy } \\
\text { (kcal/mol) }\end{array}$ & $\begin{array}{c}\text { Intermolecular } \\
\text { interaction } \\
\text { energy } \\
\text { (kcal/mol) }\end{array}$ & $\begin{array}{l}\text { vdW energy } \\
\text { (kcal/mol) }\end{array}$ & $\begin{array}{c}\text { Electrostatic } \\
\text { energy } \\
\text { (kcal/mol) }\end{array}$ \\
\hline Hit_I & CHEMBL232154 & $\mathrm{C}_{29} \mathrm{H}_{40} \mathrm{ClN}_{7} \mathrm{O}_{4}$ & 586.12 & -9.0 & 13.4 & -28.8 & -26.1 & -2.7 \\
\hline \multirow[t]{2}{*}{ Hit_II } & KWFSWQGHHVLWDM- & & & & & & & \\
\hline & UHFFFAOYSA-N & $\mathrm{C}_{24} \mathrm{H}_{30} \mathrm{~N}_{6} \mathrm{O}_{4} \mathrm{~S}$ & 498.60 & -8.2 & 3.5 & -35.3 & -22.5 & -12.9 \\
\hline Hit_III & MolPort-005-050-255 & $\mathrm{C}_{22} \mathrm{H}_{26} \mathrm{ClF}_{2} \mathrm{~N}_{3} \mathrm{O}_{2}$ & 437.91 & -8.9 & 38.1 & -36.6 & -21.0 & -15.6 \\
\hline Hit_IV & PubChem-46907406 & $\mathrm{C}_{31} \mathrm{H}_{45} \mathrm{~N}_{5} \mathrm{O}_{5}$ & 567.72 & -9.2 & 20.6 & -30.1 & -26.7 & -3.4 \\
\hline $\begin{array}{c}\text { Control } \\
\text { (BQA) }\end{array}$ & - & $\mathrm{C}_{26} \mathrm{H}_{29} \mathrm{BrF}_{3} \mathrm{~N}_{2} \mathrm{O}_{3}$ & 554.419 & -8.6 & 16.9 & -49.7 & -21.6 & -28.1 \\
\hline
\end{tabular}

surface representations and 2D interactions of BQA with PDZ domain interacting residues. It can be seen from Fig. 3 that BQA interacts with the PDZ domain through multiple hydrogen bonds and hydrophobic contacts. Hydrogen bonds are formed by the carboxylic acid and amide groups whereas hydrophobic interactions are formed by the remaining sites such as cyclopropyl ring, trifluoromethyl group, a bromine atom, and aromatic rings present in the BQA. The Binding pocket has Asp28, Leu32, Ile33, Gly34, Ile35, Phe53, Thr56, Lys83, and Ala87 residues. $\mathrm{R} 1$ (- $\mathrm{CF}_{3}$ group) of $\mathrm{BQA}$ is surrounded by Asp28, Leu32, and Thr56, R2 (- $\mathrm{C}_{6} \mathrm{H}_{4} \mathrm{Br}$ group) by Lys83 and Ala87, and R3 (cyclopropane ring, R-CH- $\left(\mathrm{CH}_{2}\right)_{2}$ ) by Ile33, Gly34, and Ile35 neighboring residues. During pharmacophore construction, the R3 hydrophobic pocket was ignored to screen the libraries.

\section{Structure-based pharmacophore modeling and virtual screening}

To screen the large number of compounds against the PICK1 PDZ domain, pharmacophore was constructed by selecting specific ligands features that interact with the specific residues of the PDZ domain. The aim was to find the best-fit hits for the PDZ domain and consequently, a maximum number of features (seven) were kept activated during the virtual screening. Pharmacophore was constructed from the previously reported crystal structure of PICK1 PDZ domain ${ }^{27}$ with a small organic molecule inhibitor (BQA). BQA has total of 13 features, aromatic-2 (1. phenyl ring $\left[\mathrm{R}-\underline{\mathrm{C}}_{6} \underline{\mathrm{H}}_{4} \mathrm{CF}_{3}\right]$ and 2. phenyl ring $[\mathrm{R}-$ $\underline{\mathrm{C}}_{6} \underline{\mathrm{H}}_{4} \mathrm{Br}$ ]), hydrogen donor-1 (amide group nitrogen [R-CONNH$\mathrm{R}]$ ), hydrogen acceptor-4 (1. amide group oxygen [R-CONH-R], 2. hydroxyl oxygen in the carboxylic acid group $[\mathrm{R}-\mathrm{C}=\mathrm{O}(\underline{\mathrm{O}} \mathrm{H})], 3$. carbonyl oxygen in the carboxylic acid group $[\mathrm{R}-\mathrm{C}=\underline{\mathrm{O}}(\mathrm{OH})]$, and 4. piperidine nitrogen $\left[\mathrm{R}_{2}-\mathrm{C}_{5} \mathrm{H}_{8} \underline{\mathrm{N}}-\mathrm{R}\right]$ ), hydrophobic-5 (1. phenyl ring $\left[\mathrm{R}-\underline{\mathrm{C}}_{6} \underline{\mathrm{H}}_{4} \mathrm{CF}_{3}\right], 2$. phenyl ring $\left[\mathrm{R}-\underline{\mathrm{C}}_{6} \underline{\mathrm{H}}_{4} \mathrm{Br}\right], 3$. bromine atom $[\mathrm{R}-$ 
A

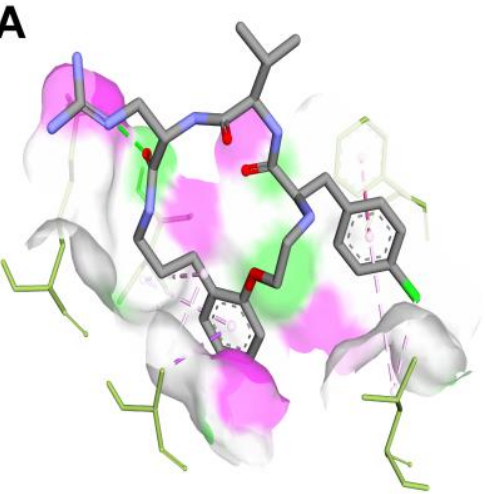

B

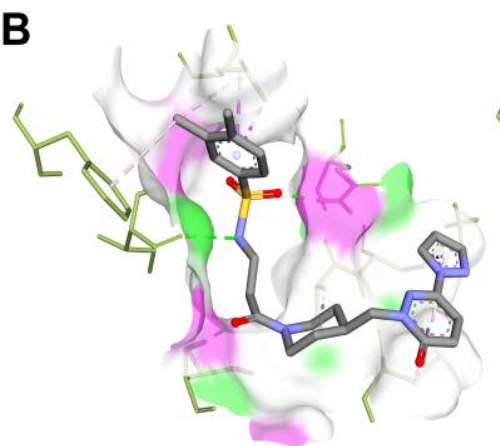

C

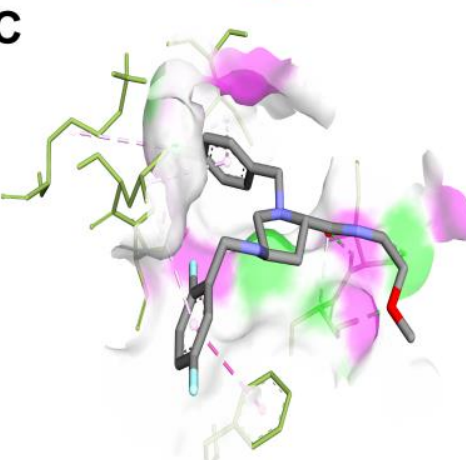

D

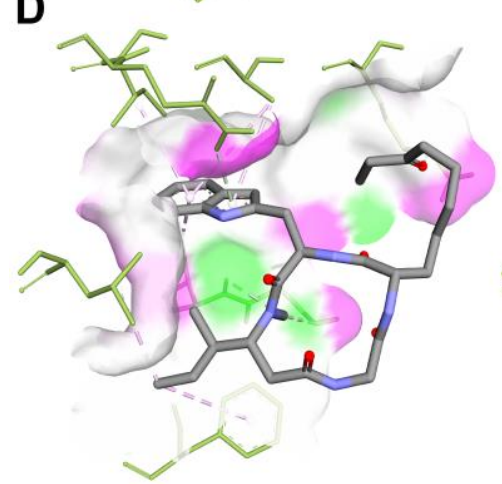

H

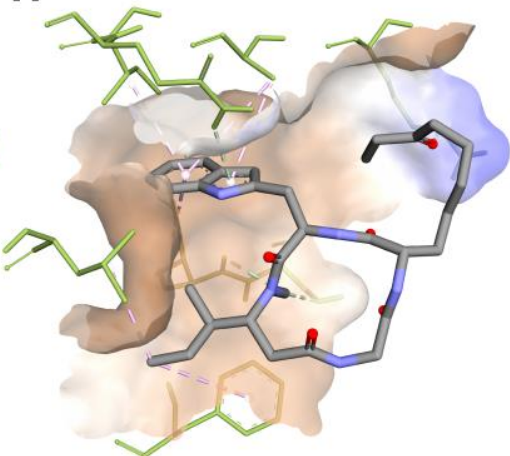

G

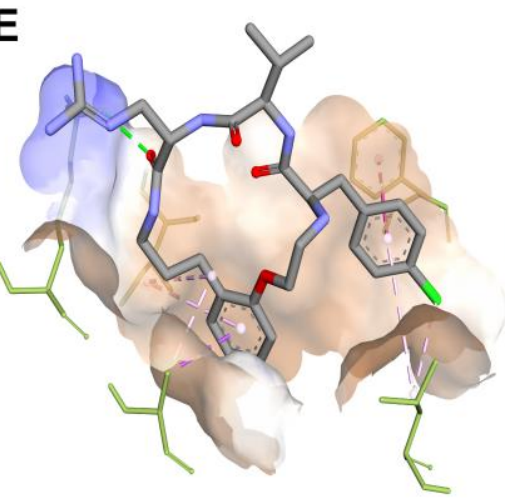

$\mathbf{F}$
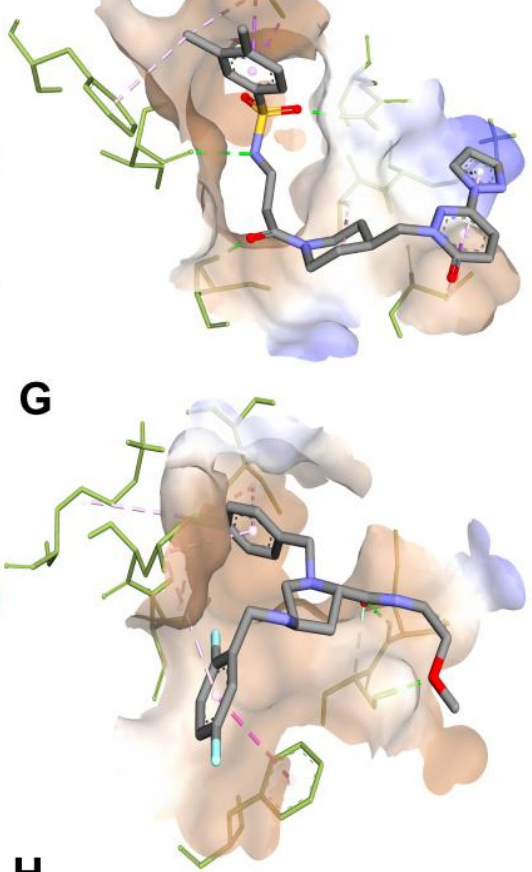

Hydrophobicity

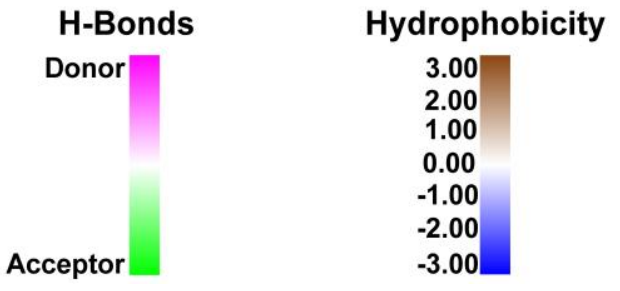

Fig. $42 \mathrm{D}$ and 3D Interactions between the hits and PDZ domain. (A-D) Hydrogen bond surface representations of Hit_I to Hit_IV respectively. (E-H) Hydrophobic surface representations of Hit_I to Hit_IV respectively, and (I-L) 2D interactions of Hit_I to Hit_IV respectively.
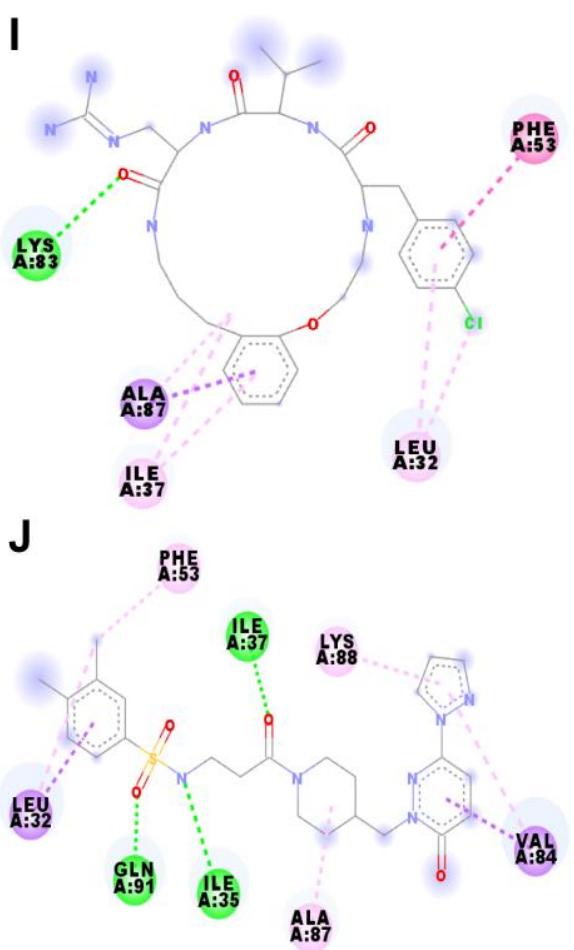

$\mathbf{K}$

PHE

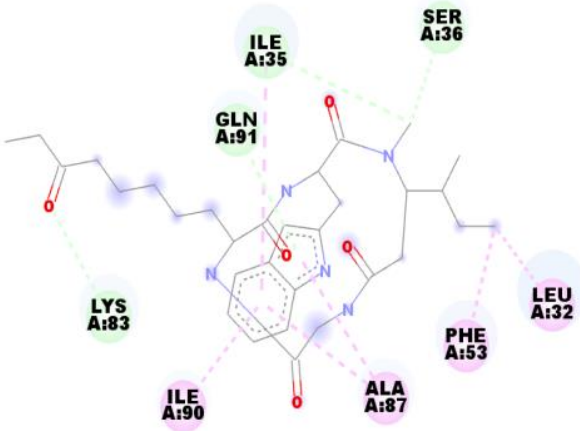

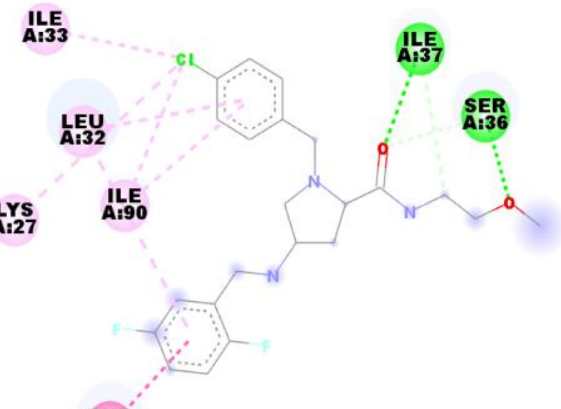


$\left.\mathrm{C}_{6} \mathrm{H}_{4} \underline{\mathrm{Br}}\right]$, 4. trifluoromethyl group [ $\left[\mathrm{R}-\mathrm{C}_{6} \mathrm{H}_{4} \mathrm{CF}_{3}\right]$, and 5. cyclopropyl ring $\left[\mathrm{R}-\underline{\mathrm{CH}}-\left(\mathrm{CH}_{2} 2_{2}\right]\right.$ ), and 5. negative ion-1 (carboxylic acid group [R- $\underline{\mathrm{COO}}-])$ but, $\mathrm{BQA}$ interacts with $\mathrm{PDZ}$ through nine features (aromatic-1, hydrogen donor-1, hydrogen acceptor-3, hydrophobic-4) excluding four features (1. aromatic- phenyl ring $\left[R-\underline{C}_{6} \underline{H}_{4} \mathrm{Br}\right], 2$. hydrogen acceptor- piperidine nitrogen $\left[\mathrm{R}_{2}-\right.$ $\left.\mathrm{C}_{5} \mathrm{H}_{8} \underline{\mathrm{N}}-\mathrm{R}\right]$, 3. hydrophobic- phenyl ring $\left[\mathrm{R}-\underline{\mathrm{C}}_{6} \underline{H}_{4} \mathrm{CF}_{3}\right]$, and 4 . negative ion- carboxylic acid group [R- $\underline{\mathrm{COO}}]$ ). However, in pharmacophore construction, seven out of total nine were considered excluding two features (1. hydrophobic- cyclopropyl ring $\left[\mathrm{R}-\mathrm{CH}-\left(\mathrm{CH}_{2} 2_{2}\right]\right.$ and 2. hydrogen acceptor- carboxylic acid group's hydroxyl oxygen $[\mathrm{R}-\mathrm{C}=\mathrm{O}(\underline{\mathrm{OH}})]$ ). Fig. 2D-E represents the pharmacophore features.

There were ten molecular libraries (Table 1) screened against the PICK1 PDZ domain and at the end, four hits (CHEMBL25-1, MCULE-ULTIMATE-1, MolPort-1, and PubChem1) were identified (Table 2). These four hits along with BQA (control) were further prepared for molecular docking study with the PDZ domain. Pharmacophore models and identified hits are shown in Fig. 2.

\section{Molecular docking}

The binding affinity and interactions of four identified hits with the PICK1 PDZ domain were probed using the flexible molecular docking DockThor web server. Additionally, the control (BQA) was also docked with the PDZ domain. The 2D and 3D molecular structures of the four hits are shown in Fig. 2F-M. DockThor calculates the total energy, intermolecular interaction energy, van der Waals energy, and electrostatic energy along with binding affinity (score). Intermolecular energy is the sum of van der Waals and electrostatic energy. Total energy comprises three terms, (1) intermolecular energy between the protein and ligand atom pairs, (2) intramolecular energy of 1-4 non-bonded atom pairs in ligand, and (3) torsional energy of ligand. Total energy indicates the rank of different binding poses of the same ligand whereas binding affinity differentiates the ligands based on their interaction energy with a specific protein.

Docking score was found approximately in the range of -8.0 to $-9.0 \mathrm{kcal} / \mathrm{mol}$ (Table 2). From the results, it was noticed that three hits have slightly higher docking scores compared to the BQA while the remaining has slightly less than the BQA docking score. Among all, Hit_IV has the highest docking score (-9.2 $\mathrm{kcal} / \mathrm{mol})$ and Hit_ll has the lowest $(-8.2 \mathrm{kcal} / \mathrm{mol})$. Further, results suggest that the intermolecular energy of BQA with PDZ is significantly higher (>15-20 kcal $/ \mathrm{mol}$ ) in comparison with four hits and the main contribution comes from electrostatic energy $(-28.1 \mathrm{kcal} / \mathrm{mol})$. Hit_I and Hit_IV have the lowest electrostatic energy contribution (approximately $-3.0 \mathrm{kcal} / \mathrm{mol}$ ) while van der Waals energy contributions are roughly in the range of -21.0 to $-26.0 \mathrm{kcal} / \mathrm{mol}$ for all the hits and BQA. The 2D and 3D interaction diagrams of studied hits with the PDZ domain are illustrated in Fig. 4.

Hit_I (CHEMBL232154) derivatives have been reported as motilin receptor inhibitors. ${ }^{51}$ Docking score of Hit_I with PDZ domain was observed $-9.0 \mathrm{kcal} / \mathrm{mol}$. In the Hit_I-PDZ complex, Lys 83 forms a hydrogen bond with carbonyl oxygen, and Phe53 interacts with the aromatic ring through pi-pi stacking. However, mainly hydrophobic interactions (pi-alkyl and alkylalkyl) were observed with the Leu32, Ile37, and Ala87 amino acids (Fig. 4I). Additionally, the chlorine atom interacts with the Leu32 and contributes to the total interaction energy. Lys 83 and Ala87 are the part of $\alpha$-helix while Ile37 is present at the $\beta$ strand region. The second compound, Hit_ll has the lowest binding affinity $(-8.2 \mathrm{kcal} / \mathrm{mol})$ among all the compounds. Thorough the literature review, it was found that this compound has not been reported as an inhibitor for any target. There were three hydrogen bonds observed between the compound and surrounding residues (Ile35, Ile37, and GIn91). Three heterocyclic moieties, pyridazine, piperidine, and pyrazole interact with the Val84, Ala87, and Lys $88 \alpha$-helix amino acids respectively (Fig. 4J). However, the remaining dialkyl substituted aromatic ring has hydrophobic interactions with Leu32 and Phe53 residues which are present at the loop region of the PDZ domain.

Next, the Hit_III was also observed to have a good binding affinity $(-8.9 \mathrm{kcal} / \mathrm{mol})$ towards the PDZ domain. From the interactions shown in Fig. $4 \mathrm{~K}$, it can be thought that multiple hydrophobic interactions of two halogenated aromatic rings with Lys27, Leu32, Ile33, Ile90, and Phe53 vicinal amino acid residues contribute significantly to the total binding energy of the compound with PDZ domain. In addition to this, it also forms two hydrogen bonds with Ser36 and Ile37 through oxygen atoms of ether and amide functional groups respectively. Only single amino acid (lle90) is located in $\alpha$-helix while remaining residues come from the $\beta$-strand and loop regions of the PDZ domain. This compound also has not been reported in the literature as an antagonist for any protein target. Lastly, Hit_IV showed the highest binding $(-9.2 \mathrm{kcal} / \mathrm{mol})$ with the PDZ domain. Like other hits, this compound also has multiple hydrophobic interactions with the proximal amino acids such as Leu32, Ile35, Phe53, Ala87, and lle90 in the binding pocket (Fig. $4 \mathrm{~L})$. The indole aromatic ring is also interacting with Gln91 through the pi-donor hydrogen bond along with other hydrophobic interactions. Furthermore, Ile35 and Ser36 residues of $\beta$-strand form slightly polar carbon-hydrogen bonds with the amide group hydrogen and Lys 83 from the $\alpha$-helix interacts through the terminal $-\mathrm{CH} 2$ - hydrogen of Lys 83 with the ketonic oxygen atom of hydrophobic alkane chain in the compound.

Lifeng Pan and his team have solved the crystal structure of the PICK1 PDZ domain with GluA1 tail peptide and indicated which residues of the PDZ domain are responsible for PZDGluA2 interaction for the internalization of AMPA receptor. ${ }^{52}$ Their interactions analysis suggests that Lys27, Ile33, Gly34, lle35, Ser36, Ile37, Lys83, Ala87, and Ile90 amino acids significantly interact with the GluA2 peptide tail and are responsible for facilitating the AMPA internalization. Isoleucine plays the dominant role to bind GluA2 peptide. Interestingly, the tested four hits in this study selectively bind with these residues and consequently, it can be assumed that these hits will prevent the binding of the GluA2 subunit at the binding pocket of the PDZ domain. 

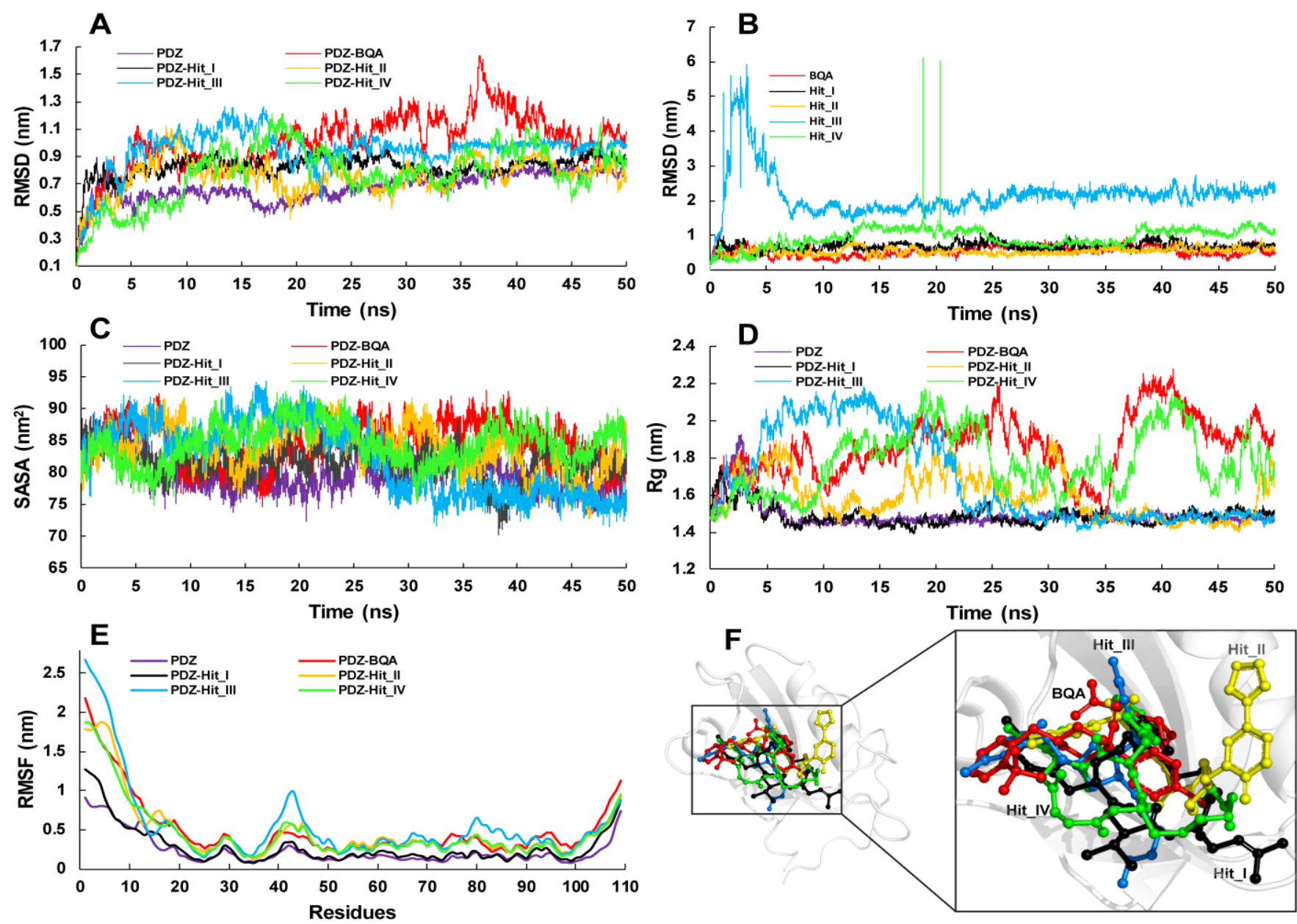

Fig. 5 MD simulations analysis of hits and control (BQA) with the PDZ domain and their docked poses at the PDZ binding pocket. (A) RMSD (nm) plots of the PDZ backbone with the ligand bound and unbound states. (B) RMSD $(\mathrm{nm})$ plots of the ligands. (C) SASA ( $\mathrm{nm}^{2}$ ) plots of PDZ with ligand bound and unbound states. (D) Rg (nm) plots of PDZ with ligand bound and unbound states. (E) RMSF (nm) plots of C-alpha atoms in PDZ with ligand bound and unbound states, and (F) Docked poses of four hits and control (BQA) inside the PDZ catalytic pocket.

\section{Molecular dynamic simulation}

\section{Root mean square deviation (RMSD)}

To get insight into the dynamicity of protein-ligand complexes, 50 ns all-atom molecular dynamic simulation was performed using the GROMACS tool. The protein conformational changes and secondary structure stability upon ligand binding were investigated by analyzing 50 ns MD trajectories for root mean square deviation (RMSD) of the protein backbone and root mean square fluctuation (RMSF) of alpha-carbon atoms of the protein. Fig. 5A and Fig. 5E represent the RMSD and RMSF plots respectively. Among all the complexes, the mean RMSD of PDZBQA was observed highest $(1.0 \mathrm{~nm})$. PDZ-Hit_III also showed significant RMSD $(0.94 \mathrm{~nm})$ while the remaining had an average $0.75 \mathrm{~nm}$ RMSD. RMSD of ligand unbound PDZ was noticed 0.67 $\mathrm{nm}$ which indicates that upon ligand binding, protein dynamicity increases significantly. Additionally, it was observed that for the initial 20 ns of simulation, the RMSD of all the complexes except PDZ-BQA were increased and then remained constant between $0.5 \mathrm{~nm}-1.2 \mathrm{~nm}$. Hence, ligand binding PDZ gains stability for the remaining simulation time. Also, tested hits give more stability to the PDZ domain in comparison with control BQA.
Furthermore, the RMSD of each ligand was also computed from the 50 ns trajectory of each complex. Fig. 5B illustrates the RMSD of four hits along with BQA. It can be seen from Fig. 5B that Hit_III showed the highest mean RMSD $(2.2 \mathrm{~nm})$ which indicates its higher dynamicity inside the protein. Hit_llI fluctuates considerably for the first 7-8 ns and then it remains stable during the last 48 ns. Hit_IV also changes its conformations significantly (average RMSD: $0.93 \mathrm{~nm}$ ) upon protein binding during the simulation compared to the remaining ones (mean RMSD: $0.55 \mathrm{~nm}$ ).

\section{Root mean square fluctuation (RMSF)}

RMSF analysis indicates how protein residues fluctuate with the ligand binding. Leaving $\mathrm{C}$ - and $\mathrm{N}$-terminal regions (C-terminal: 101-109, N-terminal: 1-20), average RMSF values of PDZ alone and ligand-bound complexes were computed. RMSF values indicate that the PDZ unbound state has the lowest RMSF $(0.15$ $\mathrm{nm}$ ) whereas PZD-Hit_III has the highest fluctuations (RMSF: $0.38 \mathrm{~nm}$ ). In the other complexes, mean RMSF was observed $0.28 \mathrm{~nm}$. Furthermore, interestingly it was observed that ligand binding residues (Lys27, Leu32, Ile33, Ile35, Ser36, Ile37, Phe53, Lys83, Val84, Ala87, Lys88, Ile90, and GIn91) do not fluctuate significantly. However, the residues, Gly40, Ala41, Gln42, Tyr43, 
A

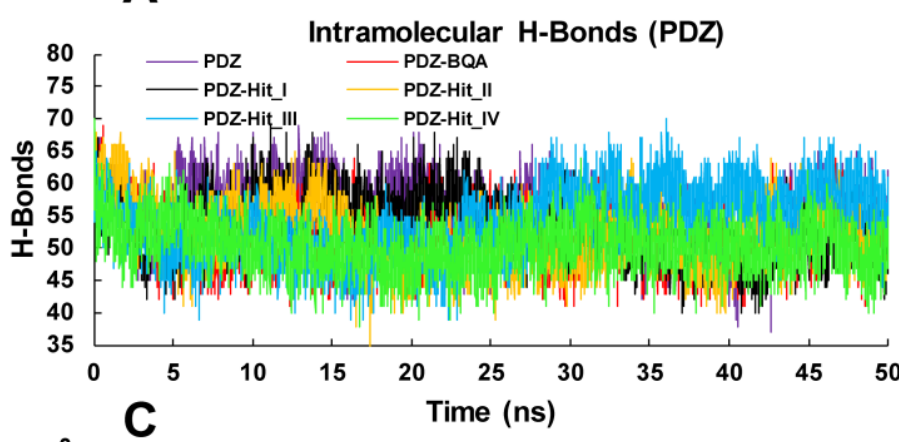

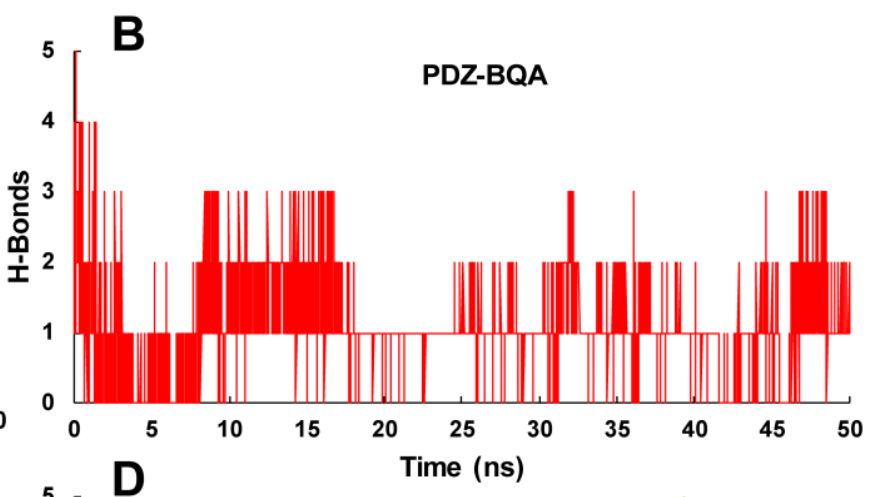

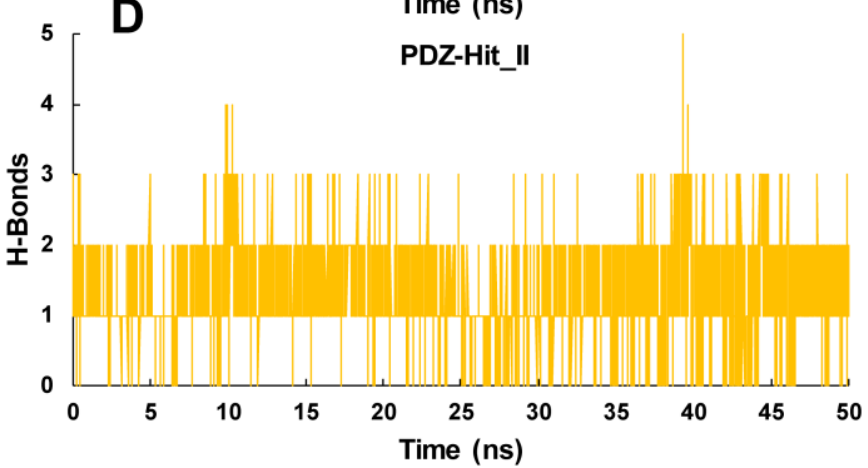

$\mathbf{F}$

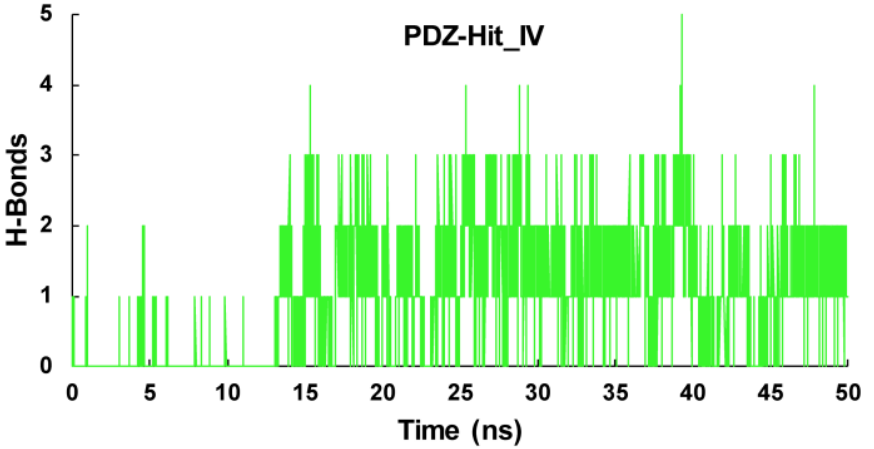

Fig. 6 MD simulation analysis of Intramolecular and intermolecular hydrogen bonds in PDZ and its complexes. (A) Intramolecular hydrogen bonds in PDZ with ligand bound and unbound states and, (B-F) Intermolecular hydrogen bonds in the complexes of PDZ with the Hit_I to Hit_IV respectively.

Cys44, Pro45, and Cys46 in the loop region connecting two betastrands and residues, Lys79, Gly80, and Thr81 in another loop connecting helix and beta-strand showed higher fluctuations.

\section{Solvent accessible surface area (SASA)}

To further understand the PDZ domain stability and conformational changes upon ligand binding, solvent accessible surface area (SASA) was determined from the 50 ns trajectory for all the complexes along with PDZ alone. The mean values were observed $79.9 \mathrm{~nm}^{2}, 84.7 \mathrm{~nm}^{2}, 81.7 \mathrm{~nm}^{2}, 83.8 \mathrm{~nm}^{2}, 82.2$ $\mathrm{nm}^{2}$, and $84.5 \mathrm{~nm}^{2}$ for PDZ, PDZ-BQA (control), PDZ-Hit_I, PDZHit_II, PDZ-Hit_III, and PDZ-Hit_IV respectively. The high SASA value indicates the structure expansion and solvent can access more surface area of protein. The mean SASA value does not give a clear indication of the influence of ligand binding on protein stability. Hence, trajectory with respect to simulation time was interpreted. From the trajectory analysis, it was observed that the SASA for the PDZ-Hit_III was reduced significantly compared to other complexes including control. Additionally, the SASA for the PDZ domain alone was also reduced at the end of the simulation. For the remaining ones, SASA was not significantly increased or decreased but fluctuated between time steps which indicates the slightly opening and closing of the PDZ domain upon ligand binding. Thus, the SASA value suggests that Hit_ll efficiently stabilizes the PDZ domain upon its binding. Fig. $5 \mathrm{C}$ represents the SASA plots.

\section{Radius of gyration (Rg)}

Protein compactness variation during the simulation can be measured by calculating the radius of gyration ( $\mathrm{Rg}$ ) of protein. The mean values of Rg of PDZ, PDZ-BQA, PDZ-Hit_I, PDZ-Hit_II, PDZ-Hit_III, and PDZ-Hit_IV were observed at $1.5 \mathrm{~nm}, 1.9 \mathrm{~nm}$, $1.5 \mathrm{~nm}, 1.6 \mathrm{~nm}, 1.7 \mathrm{~nm}$, and $1.8 \mathrm{~nm}$ respectively. There was a significant deviation in compactness observed for all the complexes except PDZ and PDZ-Hit_I (Fig. 5D). Rg values of PDZ and PDZ-Hit_I complex were increased during the first 2 ns simulation. Then, they remained constant between 1.4-1.5 nm. However, in the case of PDZ-Hit_III, Rg was observed between 1.5-2.2 $\mathrm{nm}$ in the initial $20 \mathrm{~ns}$, and then, it was quite stable (1.4- 
$1.5 \mathrm{~nm}$ ) during the remaining simulation. For PDZ-Hit_II, Rg was observed considerably less $(1.4-1.9 \mathrm{~nm})$ than the Rg values (1.5$2.3 \mathrm{~nm}$ ) of PDZ-Hit_IV and PDZ-BQA. Thus, PDZ-Hit_I, PDZ-Hit_II, and PDZ-Hit_III complexes became compact at the end of the simulation which indicates the stabilization of PDZ through ligand binding.

\section{Hydrogen bonding}

Polar interactions between the protein and ligand contribute significantly to the binding affinity between them. Conventional hydrogen bond formation between the protein and ligand stabilizes the complex. Apart from RMSD, RMSF, SASA, and Rg, intramolecular hydrogen bond formation in PDZ domain and intermolecular hydrogen bond formation between the ligand and protein were calculated from the 50 ns trajectory. Intramolecular hydrogen bonds in PDZ and intermolecular hydrogen bonds in complexes are depicted in Fig. 6A and Fig. $6 \mathrm{~B}-\mathrm{F}$ respectively. The average number of intramolecular hydrogen bonds in PDZ, PDZ-BQA, PDZ-Hit_I, PDZ-Hit_II, PDZHit_III, and PDZ-Hit_IV were identified 55, 52, 54, 52, 54, and 50 respectively. Thus, Hit_IV disrupts the intramolecular hydrogen bonds network in PDZ to a greater extent compared to other hits and control BQA. In the case of intermolecular hydrogen bonds, Hit_II, Hit_IV, and BQA form a maximum of five bonds with the protein compared to Hit_l and Hit_III which form three and two respectively. During the 50 ns simulation, hydrogen bonds between the protein and ligand were persistent in all the complexes except PDZ-Hit_III. Hence, hydrogen bonding contributes significantly to the binding energy of all complexes except PDZ-Hit_III.

\section{Conclusion}

To identify the potential hits for the PICK1 PDZ domain, pharmacophore-based virtual screening was performed using ten libraries containing 340,731,400 molecules and $1,603,779,177$ conformers. Pharmacophore was constructed using a total of seven features. There were four potent hits were identified from the different libraries. These hits were further subjected to molecular docking along with control inhibitor BQA. Docking results revealed that three hits, Hit_I, Hit_III, and Hit_IV showed a slightly higher binding affinity with the PDZ domain in comparison with control and Hit_IV was found to have the highest binding tendency $(-9.2 \mathrm{kcal} / \mathrm{mol})$ towards the PDZ domain. To probe the ligand binding and dynamic behavior of the protein-ligand complexes, molecular dynamic simulation was performed. From the RMSD, RMSF, SASA, Rg, and hydrogen bond analysis, it was found that Hit_I stabilizes the PDZ domain radically throughout the simulation compared to other hits. Additionally, simulation results also suggest that Hit_III can be considered a good hit because it also stabilizes PDZ for the last 25 ns simulation even though it forms a fewer number of hydrogen bonds with the PDZ. One possible explanation for Hit_III for PDZ stabilization is the contribution of substantial hydrophobic interactions with the PDZ domain. These are primary indications that these hits efficiently bind with the
PICK1 PDZ domain and compete with the GluA2 subunit of AMPA receptor binding at the catalytic pocket. Thus, these hits might inhibit and show potency against the PDZ domain but further experimental pieces of evidence are needed to verify this finding.

\section{Conflicts of interest}

There are no conflicts to declare.

\section{Acknowledgements}

Author is thankful to Mohmedyasin F. Mansuri from the Microbiology Department for language corrections and proofreading. Also, the author is thankful to his Chemistry Department for providing computational and infrastructure facilities.

\section{References}

1 J. Xu and J. Xia, NeuroSignals, 2007, 15, 190-201.

2 R. Herlo, V. K. Lund, M. D. Lycas, A. M. Jansen, G. Khelashvili, R. C. Andersen, V. Bhatia, T. S. Pedersen, P. B. C. Albornoz, N. Johner, I. Ammendrup-Johnsen, N. R. Christensen, S. Erlendsson, M. Stoklund, J. B. Larsen, H. Weinstein, O. Kjærulff, D. Stamou, U. Gether and K. L. Madsen, Cell Rep., 2018, 23, 2056-2069.

3 Y. H. Li, N. Zhang, Y. N. Wang, Y. Shen and Y. Wang, Neurochem. Int., 2016, 98, 115-121.

4 S. Erlendsson, M. Rathje, P. O. Heidarsson, F. M. Poulsen, K. L. Madsen, K. Teilum and U. Gether, J. Biol. Chem., 2014, 289, 25327-25340.

5 T. S. Thorsen, K. L. Madsen, N. Rebola, M. Rathje, V. Anggono, A. Bach, I. S. Moreira, N. Stuhr-Hansen, T. Dyhring, D. Peters, T. Beuming, R. Huganir, H. Weinstein, C. Mulle, K. Strømgaard, L. C. B. Rønn and U. Gether, Proc. Natl. Acad. Sci. U. S. A., 2010, 107, 413-418.

6 M. M. R. Arkin and J. A. Wells, Nat. Rev. Drug Discov., 2004, 3, 301-317.

7 J. A. Wells and C. L. McClendon, Nature, 2007, 450, 1001 1009.

8 T. Berg, Curr. Opin. Drug Discov. Devel., 2008, 11, 666-674.

9 K. K. Dev, Nat. Rev. Drug Discov., 2004, 3, 1047-1056.

10 L. L. Blazer and R. R. Neubig, Neuropsychopharmacology, 2009, 34, 126-141.

11 M. D. Houslay, Br. J. Pharmacol., 2009, 158, 483-485.

12 H. Kornau, L. T. Schenker, M. B. Kennedy and P. H. Seeburg, Science, 1995, 269, 1737-1740.

13 R. Rye, Nature, 1995, 378, 603-605.

14 K. F. Sulvan, A. R. Ceilbioi, J. D. F. F. R. Turner, E. C. Raff, D. Bioi, J. D. F. J. A. H. F, H. D. H. J. A. H. F. R. Turner, C. Raff, D. Genet, A. Gasch, U. Hnz, E. C. Raff, B. Dev, R. W. Dettman, E. C. R. Genetics, R. W. Dettman, F. R. Turner, E. C. Ray, Z. Songyang, A. S. Fanning, C. Fu, J. Xu, S. M. Marfatia, A. H. Chishti, A. Crompton, A. C. Chan, J. M. Anderson and L. C. Cantley, Science, 1997, 275, 73-77.

15 S. M. Gardner, K. Takamiya, J. Xia, J. G. Suh, R. Johnson, S. Yu and R. L. Huganir, Neuron, 2005, 45, 903-915.

16 M. I. Daw, R. Chittajallu, Z. A. Bortolotto, K. K. Dev, F. Duprat J. M. Henley, G. L. Collingridge and J. T. R. Isaac, Neuron, 2000 28, 873-886.

17 J. Xia, H. J. Chung, C. Wihler, R. L. Huganir and D. J. Linden, Neuron, 2000, 28, 499-510. 
18 A. Terashima, K. A. Pelkey, J. C. Rah, Y. H. Suh, K. W. Roche, G. L. L. Collingridge, C. J. McBain and J. T. R. Isaac, Neuron, 2008, 57, 872-882.

19 E. M. Garry, A. Moss, R. Rosie, A. Delaney, R. Mitchell and S. M. Fleetwood-Walker, Mol. Cell. Neurosci., 2003, 24, 10-22.

20 J. D. Bell, E. Park, J. Ai and A. J. Baker, Cell Death Differ., 2009, 16, $1665-1680$.

21 R. M. Dixon, J. R. Mellor and J. G. Hanley, J. Biol. Chem., 2009, 284, 14230-14235.

22 C. Bellone and C. Lüscher, Nat. Neurosci., 2006, 9, 636-641.

23 J. G. Hanley and J. M. Henley, EMBO J., 2005, 24, 3266-3278.

24 H. Hsieh, J. Boehm, C. Sato, T. Iwatsubo, T. Tomita, S. Sisodia and R. Malinow, Neuron, 2006, 52, 831-843.

25 S. Alfonso, H. W. Kessels, C. C. Banos, T. R. Chan, E. T. Lin, G. Kumaravel, R. H. Scannevin, K. J. Rhodes, R. Huganir, K. M. Guckian, A. W. Dunah and R. Malinow, Eur. J. Neurosci., 2014 39, 1225-1233.

26 S. Ducki and E. Bennett, Curr. Chem. Biology, 2009, 3, 146-158

27 E. Y. S. Lin, L. F. Silvian, D. J. Marcotte, C. C. Banos, F. Jow, T. R. Chan, R. M. Arduini, F. Qian, D. P. Baker, C. Bergeron, C. A. Hession, R. L. Huganir, C. F. Borenstein, I. Enyedy, J. Zou, E. Rohde, M. Wittmann, G. Kumaravel, K. J. Rhodes, R. H. Scannevin, A. W. Dunah and K. M. Guckian, Sci. Rep., 2018, 8, 1-10.

28 D. J. Marcotte, J. C. Hus, C. C. Banos, C. Wildes, R. Arduini, C. Bergeron, C. A. Hession, D. P. Baker, E. Lin, K. M. Guckian, A. W. Dunah and L. F. Silvian, Protein Sci., 2018, 27, 672-680.

29 A. Bach, N. Stuhr-Hansen, T. S. Thorsen, N. Bork, I. S. Moreira, K. Frydenvang, S. Padrah, S. B. Christensen, K. L. Madsen, H. Weinstein, U. Gether and K. Strømgaard, Org. Biomol. Chem. 2010, 8, 4281-4288.

30 G. Wang, X. Zhang, X. Pan and Y. Xiao, Neurosci. Lett., 2021, 741, 1-5.

31 T. S. Thorsen, K. L. Madsen, T. Dyhring, A. Bach, D. Peters, K. Stromgaard and U. Gether, Comb. Chem. High Throughput Screen., 2011, 14, 590-600.

32 N. Eswar, B. Webb, M. A. Marti-Renom, M. S. Madhusudhan D. Eramian, M. Y. Shen, U. Pieper and A. Sali, Curr. Protoc. Bioinformatics, 2006, Chapter 5, Unit-5.6.

33 J. Sunseri and D. R. Koes, Nucleic Acids Res., 2016, 44, W442W448.

34 R. López, Adv. Math. (N. Y)., 2014, 262, 476-483.

35 T. A. Halgren, J. Comput. Chem., 1996, 17, 490-519.

36 C. Chen, Y. Huang, X. Ji and Y. Xiao, J. Chem. Phys., 2013, 138, 1-9.

37 K. B. Santos, I. A. Guedes, A. L. M. Karl and L. E. Dardenne, J. Chem. Inf. Model., 2020, 60, 667-683.

38 M. J. Abraham, T. Murtola, R. Schulz, S. Páll, J. C. Smith, B. Hess and E. Lindah, SoftwareX, 2015, 1-2, 19-25.

39 D. MacKerell, D. Bashford, M. Bellott, R. L. Dunbrack, J. D. Evanseck, M. J. Field, S. Fischer, J. Gao, H. Guo, S. Ha, D. Joseph-McCarthy, L. Kuchnir, K. Kuczera, F. T. K. Lau, C. Mattos, S. Michnick, T. Ngo, D. T. Nguyen, B. Prodhom, W. E. Reiher, B. Roux, M. Schlenkrich, J. C. Smith, R. Stote, J. Straub, M. Watanabe, J. Wiórkiewicz-Kuczera, D. Yin and M. Karplus, J. Phys. Chem. B, 1998, 102, 3586-3616.

40 R. B. Best, X. Zhu, J. Shim, P. E. M. Lopes, J. Mittal, M. Feig and A. D. MacKerell, J. Chem. Theory Comput., 2012, 8, 3257-3273.

41 W. L. Jorgensen, J. Chandrasekhar, J. D. Madura, R. W. Impey and M. L. Klein, J. Chem. Phys., 1983, 79, 926-935.

$42 \mathrm{~W} . \mathrm{Yu}, \mathrm{X}$. He, K. Vanommeslaeghe and A. D. MacKerell, J. Comput. Chem., 2012, 33, 2451-2468.

43 A. Allouche, J. Comput. Chem., 2012, 32, 174-182.

44 Olivei, J. Phys. D. Appl. Phys., 1976, 9, 183-195.

45 T. Darden, D. York and L. Pedersen, J. Chem. Phys., 1993, 98, 10089-10092.
46 R. A. Lippert, K. J. Bowers, R. O. Dror, M. P. Eastwood, B. A. Gregersen, J. L. Klepeis, I. Kolossvary and D. E. Shaw, J. Chem. Phys., 2007, 126, 2006-2008.

47 H. J. C. Berendsen, J. P. M. Postma, W. F. Van Gunsteren, A. Dinola and J. R. Haak, J. Chem. Phys., 1984, 81, 3684-3690.

48 M. Parrinello and A. Rahman, J. Appl. Phys., 1981, 52, 7182 7190.

49 Hess, H. Bekker, H. J. C. Berendsen and J. G. E. M. Fraaije, J. Comput. Chem., 1997, 18, 1463-1472.

50 H. Kim, Hee Jung Chung, H. K. Lee and R. L. Huganir, Proc. Natl. Acad. Sci. U. S. A., 2001, 98, 11725-11730.

51 Marsault, K. Benakli, S. Beaubien, C. Saint-Louis, R. Déziel and G. Fraser, Bioorganic Med. Chem. Lett., 2007, 17, 4187-4190.

52 L. Pan, H. Wu, C. Shen, Y. Shi, W. Jin, J. Xia and M. Zhang, EMBO J., 2007, 26, 4576-4587. 